\title{
The expanding HIV-1 epidemic aggravates therapeutics and vaccine development programs in India
}

\author{
Ujjwal Neogi \\ Infectious Disease Unit, St. John’s Research Institute, Koramangla, Bangalore-560034; Ujjwal Neogi - Email: ujjwalneogi@gmail.com;
}

Received July 10, 2009; Accepted July 25, 2009; Published September 30, 2009

\begin{abstract}
s:
Human Immunodeficiency Virus Type 1 (HIV-1) displays extensive genetic diversity globally that poses a serious challenge in designing suitable therapeutic measures for protective immune responses in the host. After the first detection of AIDS cases in 1986, India now has the third largest HIV epidemic in world. The HIV-1 epidemic in India is still growing with introduction of new strains/subtypes and this creates a serious problem for the clinician and basic science researchers to find the effective measures. Neighboring countries in the North and North-eastern regions play an important role in the HIV-1 pandemic. An extensive multinational collaborative research effort is needed in order to identify the risk groups and geographic locales is required to monitor HIV-1 spread in Asia and to produce a successful preventive program.
\end{abstract}

Key words: HIV-1 Epidemiology, India, Asia, vaccine, therapeutics, co-operation.

\section{Background}

The first case of HIV infection and AIDS was documented in India in 1986, within five years of the first description of AIDS in North America. The progress of the HIV epidemic was relatively slow, initially. Over the years, the number of HIV infected persons increased and it is a major public health problem now. The estimated number of HIV-1 infected individuals in India was 2.5 million in 2008, which placed India at third place behind South Africa and Nigeria [1].

\section{HIV-1 Genetic Diversity in India}

The expanding nature of the HIV-1 epidemic and introduction of Circulating Recombinant Forms (CRFs) and Unique Recombinant Forms (URFs) along with subtypes in addition to the predominant Subtype C, poses a serious clinical and public health concern in India from the very beginning of this pandemic. The genetic analysis of HIV-1 from different parts of India reveals Subtype C strains as a predominant. However, the introduction of other subtypes in high risk group HIV-1 infected patients, primarily female commercial sex workers (CSWs) and intravenous drug users (IDUs), created challenges for therapy and vaccine development programs. A diversified HIV-1 pandemic has been found in different regions of India. The South Indian epidemic is mostly conserved in nature; although, there is the co-existence of Subtypes C, B and CRF08_BC. A group lead by Dr. Udaykumar Ranga at Jawaharlal Nehru Centre for Advance Scientific Research, also found novel B/C recombinant viruses in these populations that are not represented elsewhere.

The most diverse pattern was observed in the North and North-eastern regions of India. Work in the North East by Dr. Sekhar Chakrabarti and his colleagues at the National Institute of Cholera and Enteric Disease, Kolkata and National AIDS Research Institute (NARI), Pune, lead by Dr. Ramesh Paranjape, revealed the presence of Subtype C, Thai B, B and novel B/C recombinants of different ancestral origins in CSWs and IDUs. The reports from Maharastra, a south-western state also found diverse patterns. The highest divergent strains were found in Northern region from the initial stage of its characterizations. Reports from Dr. Shahid Jameel at International Centre for Genetic Engineering and Biology, Dr. Pradeep Seth at All India Institute of Medical Science, and their co-workers found different subtypes including C, A, B, and E in North India. Recently Dr. Akhil Banerjea and his group at the National Institute of Immunology have reported intra- and inter-B/C recombinants with similarity to corresponding strains in China. According to several Indian researchers, the prevalence of other predominant HIV-1 subtypes in neighboring countries primarily including Pakistan, China, Thailand and Myanmar, plausibly play important determinative factors in HIV-1 epidemiology in India [2,3]. Co-circulation of different subtypes in high risk populations mainly among CSWs and IDUs opens a wide array of chances of generation of intra- and inter-recombinant strains in India. Recombinant strains already pose serious global health problems in countries such as China and on the African continent.

\section{Vaccine research}

At the risk of losing the battle to control AIDS with slogans, condoms, foreign grants from World Bank, National Institute of Health, USA (check numbers of grants on http://crisp.cit.nih.gov/), and Bill and Melinda Gates Foundation (BMGF), the Indian government in 1998 launched a program to develop a vaccine against the disease [4]. In India, the first vaccine trial was initiated at NARI Pune, in 2005 and was completed in January 2007. This was a Phase I study of the Adeno-Associated Virus (AAV) based HIV-1 subtype C vaccine tgAAC09. It is a non-pathogenic virus often found in people infected with adenoviruses, genetically modified with three HIV-1 subtype C Gag, Protease and part of the reverse transcriptase gene. The second phase I trial was recently completed at the Tuberculosis Research Center (TRC) with TBC-M4; a modified Vaccinia Ankara (MVA) based vaccine. The International AIDS Vaccine Initiative (IAVI) and biotechnology firm Therion Biologics Corporation (Cambridge, Massachusetts, USA) with the assistance of Dr. Sekhar Chakrabarty, an Indian Scientist from the NICED, Kolkata, designed the candidate and manufactured doses of this vaccine for initial clinical trials. This 'modified vaccinia Ankara (MVA) vaccine' is a genetic modified vaccinia Ankara, a harmless version of pox virus, incorporated with genetic material from six HIV genes (env, pol, gag, rev, nef and tat) from an Indian isolate of subtype C [5]. 62 people have been enrolled in these trials and the vaccines have been found to be safe and well tolerated. Expanding on the results and success of the previous trials in India, a new Phase I trial is being considered in both NARI and TRC [6].

\section{Challenges and Possible Solutions}

The genetic hypervariability of HIV-1, persistence of latent integrated proviral DNA, differential transmission route, limited utility and relevance of animal models, time consuming and costly clinical trials among motivated volunteer participants, poses unprecedented scientific and organizational challenges to develop vaccine and therapeutic success against this disease. As the transmission routes of several HIV-1 strains have been traced among several neighboring countries, an active scientific coordination is needed between these countries in order to identify the risk groups and geographic locales where new CRFs or multidrug-resistant HIV-1 strains occur and may emerge further [7]. National level vigorous research on HIV-1 genetics in effective collaboration with clinical and basic science researchers is essential for successful and effective therapeutics for all patients suffering from this world's deadly disease. 


\section{Bioinformation}

Acknowledgement

I would like to thank my "Scientific Guru”, Dr. Akhil C Banerjea, for giving me opportunity to work under his guidance. And I would also like to thank Dr. Anura V Kurpad; Dean, Dr. Anita Seth; Associate

Professor, St. John's Research Institute and Dr. Ayesha de Costa, Karolinska Institute, Sweden for offering me SRF for HIVIND project.
References

[1] http://www.unaids.org

[2] U Neogi et al., Indian J Exp Biol. 57:424 (2009)

[3] S Lakhashe et al., J Biosci. 33:515 (2008) [PMID: 19208977]

[4] DR Arora et al Indian J Med Microbiol. 21:225 (2003)

[5] http://www.iavi.org.in/vacc_devlpt_india.html

[6] http://www.nari-icmr.res.in/

[7] K A Lau AIDS Reviews 9:218 (2007) [PMID: 18219365]

Edited by P. Shapshak

Citation: Neogi, Bioinformation 4(3): 90-91 (2009) License statement: This is an open-access article, which permits unrestricted use, distribution, and reproduction in any medium, for noncommercial purposes, provided the original author and source are credited. 